\title{
A NEW INDEX FOR THE INTENSITY OF NATURAL SELECTION
}

\author{
B. F. J. MANLY \\ Biometrics Unit, University of Otago, P.O. Box 56, Dunedin, New Zealand
}

Received 8.x.76

\section{Summary}

A new index for the intensity of natural selection is proposed, based upon the double exponential model for fitness functions. This index is defined as a variance and is such that a value of zero indicates no selection while a value of one indicates quite strong selection. The use of the index is demonstrated using published data on the survival of human infants with different birth weights and gestation times.

\section{INTRODUGTION}

IN an earlier paper (Manly, 1976) I suggested that a double exponential fitness function provides a useful model for the analysis of certain types of survival data. With this model the probability of an individual surviving natural selection over a time period $(0, t)$ is given by the equation

$$
p_{t}=\exp \left\{-g(t) \exp \left(\alpha_{0}+\alpha_{1} X_{1}+\ldots+\alpha_{m} X_{m}\right)\right\}
$$

where $g(t)$ is a positive, non-decreasing function of time such that $g(0)=0$. In some cases $g(t)$ might simply equal $t$. The $X$ 's in the equation represent characters that the probability of survival is related to (or possibly powers and products of such characters).

If equation ( 1 ) holds for all the individuals in a population then the amount of selection that these individuals are subjected to depends upon the amount of variation that there is for the linear combination

$$
f=\alpha_{1} X_{1}+\alpha_{2} X_{2}+\ldots+\alpha_{m} X_{m} .
$$

If all the individuals have the same $f$ value then there will be no selection at all. However if $f$ is very variable then the selection will be intense. This suggests that the variance of $f$, say $V_{f}$, might be a useful measure of the intensity of selection.

\section{2. $V_{f}$ AS A MEASURE OF THE INTENSITY OF SELEGTION}

As the selection period $t$ increases, so will the function $g(t)$ in equation (1). However $f$ values will remain unchanged, given that the $\alpha$ coefficients remain constant. One important property of $V_{f}$ is therefore that the length of the selection period is irrelevant providing that the measure is calculated using the population of individuals alive at the start of the period.

Another important property of $V_{f}$ is that values for it can be interpreted without a detailed consideration of the population that selection is operating on. For example $V_{f}=0$ indicates no selection at all, $V_{f}=0.01$ indicates a very low level of selection, $V_{f}=1$ indicates a high level of selection, and $V_{f}=6$ indicates an extremely high level of selection.

These interpretations for $V_{f}$ values are based upon the following calculations. Suppose that before selection starts the $f$ values are normally 


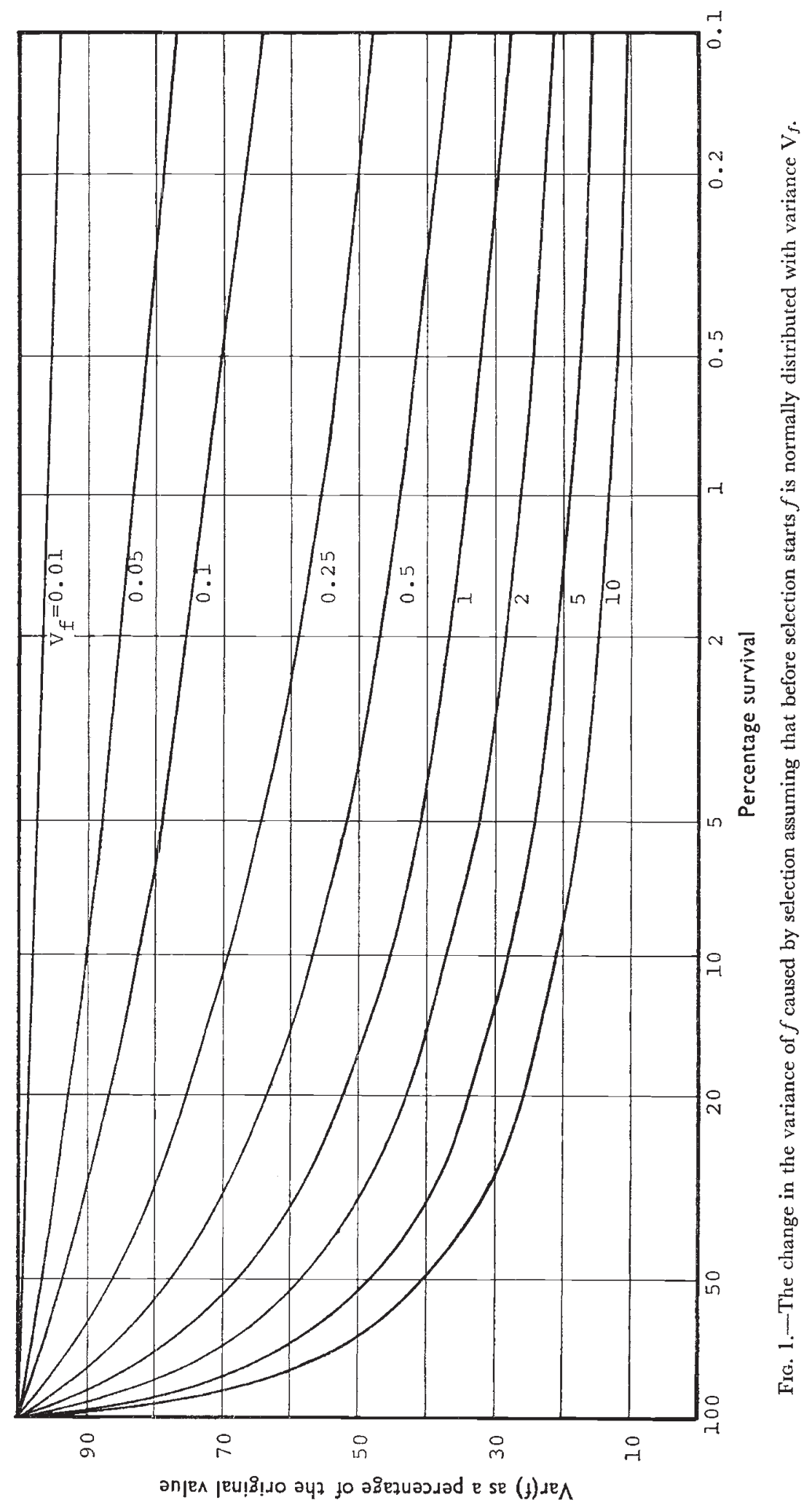


distributed within a population with standard deviation $\sigma_{f}=V_{f}^{\frac{1}{f}}$. Then approximately 95 per cent of the $f$ values will lie within the range given by the mean $\pm 2 \sigma_{f}$. Thus, after selection has operated for a time $t, 95$ per cent of survival probabilities will lie within a range of the form

$$
R=\exp \left\{-\exp \left(c_{t}-2 \sigma_{f}\right)\right\}-\exp \left\{-\exp \left(c_{t}+2 \sigma_{f}\right)\right\} .
$$

Here $c_{t}$ is a value that will depend upon $t$ and the mean of $f$. It is a simple matter to show that $R$ is at a maximum when

$$
\exp \left(c_{t}\right)=4 \sigma_{f} /\left\{\exp \left(2 \sigma_{f}\right)-\exp \left(-2 \sigma_{f}\right)\right\}
$$

where this maximum is

$$
\begin{aligned}
& R_{\max }=\exp \left[-4 \sigma_{f}\right.\left.\exp \left(-2 \sigma_{f}\right) /\left\{\exp \left(2 \sigma_{f}\right)-\exp \left(-2 \sigma_{f}\right)\right\}\right] \\
&-\exp \left[-4 \sigma_{f} \exp \left(2 \sigma_{f}\right) /\left\{\exp \left(2 \sigma_{f}\right)-\exp \left(-2 \sigma_{f}\right)\right\}\right] .
\end{aligned}
$$

Thus, for example, if $\sigma_{f}=1$ then the 95 per cent range for survival proba-

\begin{tabular}{|c|c|c|c|c|}
\hline$\sigma_{f}$ & $V_{f}$ & $R_{\max }$ & $\begin{array}{l}\text { Lower } \\
\text { prob. }\end{array}$ & $\begin{array}{l}\text { Upper } \\
\text { prob. }\end{array}$ \\
\hline 0 & 0 & 0 & - & - \\
\hline $0 \cdot 1$ & 0.01 & $0 \cdot 146$ & 0.297 & 0.443 \\
\hline $0 \cdot 2$ & 0.04 & $0 \cdot 287$ & 0.234 & 0.521 \\
\hline 0.5 & $0 \cdot 25$ & 0.632 & $0 \cdot 099$ & 0.731 \\
\hline $1 \cdot 0$ & 1.00 & 0.911 & 0.017 & 0.928 \\
\hline $1 \cdot 5$ & $2 \cdot 25$ & 0.983 & $0 \cdot 002$ & 0.985 \\
\hline $2 \cdot 0$ & $4 \cdot 00$ & $0 \cdot 997$ & $0 \cdot 0003$ & 0.997 \\
\hline $2 \cdot 5$ & $6 \cdot 25$ & 0.9995 & $0 \cdot 00005$ & 0.9995 \\
\hline
\end{tabular}
bilities will be at its widest when it is $(0.017,0.928)$, indicating a high level of selection. Table 1 was constructed using equation (3).

TABLE 1

Selection will, of course, change the distribution of $f$ since individuals with large $f$ values will have low survival probabilities while individuals with small $f$ values will have high survival probabilities. In fact the mean and variance of $f$ will both decrease for the survivors as the selection period increases. Fig. 1 shows how the variance of $f$ for survivors will change in a population for which $f$ is normally distributed before selection starts. Thus, for example, it can be seen that if the variance of $f$ is initially $1 \cdot 0\left(=V_{f}\right)$ then it will be reduced to about 0.48 by the time that 90 per cent of the population has died and further reduced to about 0.34 when 99 per cent of the population has died.

In practice $f$ values are not likely to be normally distributed. Nevertheless fig. 1 and table 1, given above, do provide a good indication of the amount of selection that is implied by a given value for $V_{f}$.

\section{The estimation of $V_{f}$}

Consider a population of $N$ individuals where the $i$ th of these has the value $x_{i j}$ for the character $X_{j}(j=1,2, \ldots, m)$. Let $\bar{x}_{j}$ denote the population mean for $X_{j}$. Then the $f$ value for the $i$ th individual will be

$$
f_{i}=\alpha_{1} x_{i 1}+\alpha_{2} x_{i 2}+\ldots+\alpha_{m} x_{i m}
$$


so that the variance will be

$$
\begin{aligned}
V_{f} & =\sum_{i=1}^{N}\left\{\alpha_{1}\left(x_{i 1}-\bar{x}_{1}\right)+\alpha_{2}\left(x_{i 2}-\bar{x}_{2}\right)+\ldots+\alpha_{m}\left(x_{i m}-\bar{x}_{m}\right)\right\}^{2} / N \\
& =\sum_{r=1}^{m} \sum_{s=1}^{m} \alpha_{r} \alpha_{s} u_{r s}
\end{aligned}
$$

where $u_{r s}$ denotes the covariance between $X_{r}$ and $X_{s}$ if $r \neq s$ and the variance of $X_{r}$ if $r=s$.

If only estimates $\hat{\alpha}_{1}, \hat{\alpha}_{2}, \ldots, \hat{\alpha}_{m}$ of the $\alpha$ 's are available then there is only an estimate

$$
\hat{V}_{f}=\sum_{r=1}^{m} \sum_{s=1}^{m} \hat{\alpha}_{r} \hat{\alpha}_{s} u_{r s}
$$

available for $V_{f}$. It is shown in the Appendix to this paper that the bias in $\hat{V}_{f}$ is given by

$$
\operatorname{Bias}\left(\hat{V}_{f}\right)=\sum_{r=1}^{m} \sum_{s=1}^{m} c_{r s} u_{r s}
$$

where $c_{r s}$ denotes the covariance between $\hat{\alpha}_{r}$ and $\hat{\alpha}_{s}$. It is also shown in the Appendix that the variance of $\hat{V}_{f}$ is

$$
\operatorname{Var}\left(\hat{\nabla}_{f}\right)=2 \sum_{i=1}^{m} \sum_{j=1}^{m} u_{i j} \sum_{r=1}^{m}\left(2 \alpha_{i} \alpha_{r}+c_{i r}\right) \sum_{s=1}^{m} u_{r s} c_{j s}
$$

assuming that the sampling errors in the $\hat{\alpha}$ 's are normally distributed with zero means.

\section{EXAmples}

There is a considerable body of literature on the survival of human infants related to their birth weights and gestation times. For example Gibson and McKeown (1951) studied this relationship for all babies born in Birmingham in 1947 and at about the same time Karn and Penrose (1951) carried out a similar study on the basis of information obtained from all the births at University College Hospital over the period 1935-46. More recently Rantakallio (1969) has investigated the situation in North Finland in 1966.

Using the computer program GLIM (Nelder, 1974; Manly, 1976) double exponential fitness functions were fitted to the data published by the authors mentioned above in order to relate the perinatal survival probability $(p)$ to birth weight $(B$, in kilograms) and gestation time $(G$, in weeks). The following functions were found to give quite good fits:

for Gibson and McKeown data:

$$
\begin{aligned}
p=\exp \{-\exp (18 \cdot 24-1.649 B-0.8190 G & +0.4093 B^{2} \\
& \left.\left.-0.05732 B G+0.01204 G^{2}\right)\right\} ;
\end{aligned}
$$

for Karn and Penrose data:

$$
\begin{aligned}
& p=\exp \{-\exp (26.85-3 \cdot 867 B-1 \cdot 152 G+ 0.7763 B^{2} \\
&\left.\left.-0.04330 B G+0.01587 G^{2}\right)\right\} ;
\end{aligned}
$$


for Rantakallio data :

$$
\begin{aligned}
p=\exp \{-\exp (11 \cdot 49+0 \cdot 3766 B-0.6365 G & +0 \cdot 7179 B^{2} \\
-0 & \left.\left.-1655 B G+0 \cdot 01383 G^{2}\right)\right\} .
\end{aligned}
$$

These equations are of the form of equation (1) with $f$ values being linear in $B, G, B^{2}, B G$ and $G^{2}$.

One obvious question that can be asked is whether equations (8) to (10) suggest similar intensities of selection. This can be answered on the basis of $V_{f}$ values but there is a complication because the bivariate distribution of birth weight and gestation time before selection was not the same for the three populations being considered. This complication can be overcome by defining a reference population with a given distribution for birth weight and gestation time and then calculating the $V_{f}$ values that such a population would have if it were subjected to the fitness functions (8) to (10). For example the reference population could be the babies born in Birminghain in 1947, as studied by Gibson and McKeown, in which case equations (8) to (10) give $V_{f}$ values of $0.76,0.46$ and $1 \cdot 09$, respectively.

In practice it does not seem to matter much which population is used as the reference population. This is shown by table 2 which gives the $V_{f}$

\section{TABLE 2}

Estimated $V_{f}$ values from

\begin{tabular}{lccc}
\cline { 2 - 3 } \multicolumn{1}{c}{ Reference population } & equation (8) & equation $(9)$ & equation (10) \\
Gibson and McKeown & $0.76( \pm 0.08)$ & $0.46( \pm 0.03)$ & $1.09( \pm 0.13)$ \\
Karn and Penrose & $0.75( \pm 0.07)$ & $0.47( \pm 0.03)$ & $1.12( \pm 0.13)$ \\
Rantakallio & $0.69( \pm 0.08)$ & $0.42( \pm 0.03)$ & $0.93( \pm 0.13)$
\end{tabular}

values that have been found using the population studied by (i) Gibson and McKeown, (ii) Karn and Penrose and (iii) Rantakallio as the reference population. The values given in parenthesis $(e . g . \pm 0.08)$ are the standard errors for the estimated values, as calculated from equation (7). Biasses are negligible.

It seems from the $\hat{V}_{f}$ values just given that the intensity of selection was lowest for the babies born at University College Hospital during the years 1935-46 and highest for those born in North Finland in 1966. This contrasts with the perinatal mortality rates of 4.5 per cent for the $1935-46$ data, 4.0 per cent for the 1947 data and 2.4 per cent for the 1966 data. An examination of the distributions of the $f$ values suggests that the reason for the higher intensity of selection with the more recent data is that the reduction in the perinatal mortality rate was due mainly to an increased survival probability for babies for which this probability was already very high.

It turns out that most of the variation in $f$ values can be related to birth weight alone. This is shown by the estimates of $V_{f}$ values in table 3 . These estimates are based upon fitted quadratic fitness functions of the form of equations (8) to (10). Rantakallio's North Finland population was used as the reference population.

Because the intensity of selection related to birth weight alone is so high the data given by Gibson and McKeown on the survival to 1 day, 1 week, 
TABLE 3

Estimated $V_{f}$ values from

Selection related to

Birth weight only

Gestation time only

Birth weight and

gestation time

$\begin{array}{ccc}\begin{array}{c}\text { Gibson and } \\ \text { McKeown data }\end{array} & \begin{array}{c}\text { Karn and } \\ \text { Penrose data }\end{array} & \begin{array}{c}\text { Rantakallio } \\ \text { data }\end{array} \\ 0.68( \pm 0.09) & 0.36( \pm 0.02) & 0.91( \pm 0.15) \\ 0.34( \pm 0.03) & 0.30( \pm 0.02) & 0.56( \pm 0.08) \\ 0.69( \pm 0.08) & 0.42( \pm 0.03) & 0.93( \pm 0.13)\end{array}$

1 month, 6 months, 9 months and 1 year after birth for infants with different birth weights has been analysed. This has been done by fitting a fitness function of the form $p=\exp \left\{-\exp \left(\alpha_{0}+\alpha_{1} B+\alpha_{2} B^{2}\right)\right\}$ separately to each of the survival periods using the computer program GLIM. (There was clear evidence that the $\alpha$ coefficients varied from period to period.) The initial population of live-born babies was then used as the reference population for calculating a $\hat{V}_{f}$ value for each survival period, with the following results (table 4):

TABLE 4

\begin{tabular}{llc}
\multicolumn{1}{c}{ Survival period } & $\hat{V}_{f}$ & Std. err. \\
Up to 1 day & 0.98 & 0.27 \\
1 day to 1 week & 0.61 & 0.14 \\
1 week to 1 month & 0.30 & 0.09 \\
1 month to 6 months & 0.17 & 0.04 \\
6 months to 9 months & 0.11 & 0.06 \\
9 months to 1 year & 0.15 & 0.08
\end{tabular}

This shows very clearly how the intensity of selection related to birth weight declined as the age of infants increased.

A referee has pointed out that these calculations on the relationship between infant survival and birth weight and gestation time are difficult to interpret because it is not possible to separate causes and effects. For example a premature baby might have a low survival probability just because it is premature or alternatively the prematurity might itself be caused by some complication of pregnancy such as toxaemia which is the real cause of the lower survival probability. However, it can be argued that birth weight and gestation time are used in practice to determine which babies have a high risk of mortality (e.g. see Battaglia and Lubchenco, 1967; Yerushalmy, 1967) and that, therefore, selection related to these variables is of some interest irrespective of what causes what.

\section{OTHER MEASURES OF THE INTENSITY OF SELEGTION}

In an earlier paper (Manly, 1975) I suggested that the measures of the intensity of natural selection proposed by Haldane (1953) and Van Valen (1965) are not satisfactory because they depend upon the length of the selection period even when the relative fitnesses of different individuals remain constant. Another index that was used by Fraccaro (1954) also suffers from this disadvantage.

In the earlier paper I also proposed an index of selective intensity that 
amounts essentially to the variance of $\exp (f)$ values scaled so that their mean is 1 , using a reference population for which all the possible $f$ values are equally frequent. One problem with this index is that if survival is being related to a continuous variable such as birth weight then there are an infinite number of possible $f$ values and the ones used in calculating the index will depend upon the arbitrary grouping of the data. Thus it seems that the use of my earlier index should be restricted to situations where the possible $f$ values really can be enumerated (e.g. see Manly, 1975, Example 2). The index proposed in the present note seems to be definitely more appropriate when a double exponential fitness function can be assumed.

Acknowledgments.-I would like to thank two referees for their helpful comments on an earlier version of this paper.

\section{REFERENGES}

Anderson, T. w. 1958. An Introduction to Multivariate Statistical Analysis. Wiley, New York. BATTAglia, F. C., AND LubChENCO, L. o. 1967. A practical classification of newborn infants by birth weight and gestational age. F. Pediat., 71, 159-163.

FRACCARO, M. 1956. A contribution to the study of birth weight based on an Italian sample. Ann. Hum. Genet., 20, 282-298.

GIBSON, J. R., AND MCKEOWN, T. 1951. Observations on all births $(23,970)$ in Birmingham, 1947. III. Survival. Brit. 7. Soc. Med., 5, 177-183.

haldane, J. B. s. 1953. The measurement of natural selection. Proc. 9th Int. Cong. Genetics, 480-487. (Carylogia, vol. suppl., 1954).

KARN, M. N., AND PENROSE, L. S. 1951. Birth weight and gestation time in relation to maternal age, parity and infant survival. Ann. Eugen., 16, 145-164.

MANLY, B. F. J. 1975. The measurement of the characteristics of natural selection. Theor. Popul. Biol., 7, 288-305.

MANLy, B. F. J. 1976. Some examples of double exponential fitness functions. Heredity, 36, 229-234.

NELDER, J. A. 1974. GLIM Manual. Distributed by the Numerical Algorithms Group, 13 Banbury Road, Oxford OX2 6NN.

RANTAKAllio, P. 1969. Groups at risk in low birth weight infants and perinatal mortality. Acta Paediat. Scand. Supp., 193.

VAN VALEN, L. 1965. Selection in natural populations. III. Measurement and estimation. Evolution, 19, 514-528.

YERUSHALMY, J. 1967. The classification of newborn infants by birth weight and gestational age. 7. Pediat., 71, 164-172.

\section{Appendix}

Let $\delta \alpha_{r}=\hat{Q}_{r}-\alpha_{r}$, the sampling error in the estimate $\hat{\alpha}_{r}$. Also let $u_{i j}$ be the covariance between $X_{i}$ and $X_{j}$ and $c_{i j}$ the covariance between $\hat{\alpha}_{i}$ and $\hat{\alpha}_{j}$ $\left(u_{i i}\right.$ and $c_{i i}$ being variances). Then from equation (5)

$$
\hat{V}_{f}=\sum_{r=1}^{m} \sum_{s=1}^{m}\left(\alpha_{r} \alpha_{s}+2 \alpha_{r} \delta \alpha_{s}+\delta \alpha_{r} \delta \alpha_{s}\right) u_{r s}
$$

and taking expected (mean) values gives

$$
E\left(\hat{V}_{f}\right)=V_{f}+\sum_{r=1}^{m} \sum_{s=1}^{m} c_{r s} u_{r s}
$$

since $E\left(\delta \alpha_{r} \delta \alpha_{s}\right)=c_{r s}$. It is being assumed here that the $\delta \alpha$ 's have expected values of zero. Thus the bias in $\hat{V}_{f}$ is given by equation (6) of this paper. 
Using equation (la)

$$
\begin{aligned}
\left(\hat{V}_{f}-V_{f}\right)^{2}= & \sum_{r=1}^{m} \sum_{s=1}^{m}\left\{\left(2 \alpha_{r} \delta \alpha_{s}+\delta \alpha_{r} \delta \alpha_{s}\right) u_{r s}\right\}^{2} \\
= & \sum_{i=1}^{m} \sum_{j=1}^{m} \sum_{r=1}^{m} \sum_{s=1}^{m}\left(4 \alpha_{i} \delta \alpha_{j} \alpha_{r} \delta \alpha_{s}+2 \alpha_{i} \delta \alpha_{j} \delta \alpha_{r} \delta \alpha_{s}\right. \\
& \left.\quad+2 \delta \alpha_{i} \delta \alpha_{j} \alpha_{r} \delta \alpha_{s}+\delta \alpha_{i} \delta \alpha_{j} \delta \alpha_{r} \delta \alpha_{s}\right) u_{i j} u_{r s} .
\end{aligned}
$$

If the $\delta \alpha$ 's are normally distributed, which they should approximately be if the $\hat{\alpha}$ 's are maximum likelihood estimators, then it is well known (e.g. see Anderson, 1958, p. 39) that

and

$$
E\left(\delta \alpha_{i} \delta \alpha_{j} \delta \alpha_{r}\right)=0
$$

$$
E\left(\delta \alpha_{i} \delta \alpha_{j} \delta \alpha_{r} \delta \alpha_{s}\right)=c_{i j} c_{r s}+c_{i r} c_{j s}+c_{i s} c_{j r} .
$$

Assuming that these results hold, taking expected values on both sides of equation (3a) gives

$E\left(\hat{\nabla}_{f}-V_{f}\right)^{2}=\sum_{i=1}^{m} \sum_{j=1}^{m} \sum_{r=1}^{m} \sum_{s=1}^{m}\left(4 \alpha_{i} \alpha_{r} c_{j s}+c_{i j} c_{r s}+c_{i r} c_{j s}+c_{i s} c_{j r}\right) u_{i j} u_{r s}$

Finally the variance of $\hat{V}_{f}$ is given by $E\left(\hat{V}_{f}-V_{f}\right)^{2}-\left\{\operatorname{Bias}\left(\hat{V}_{f}\right)\right\}^{2}$, which from equations (2a) and (4a) is

$$
\operatorname{Var}\left(\hat{V}_{f}\right)=\sum_{i=1}^{m} \sum_{j=1}^{m} \sum_{r=1}^{m} \sum_{s=1}^{m}\left(4 \alpha_{i} \alpha_{r} c_{j s}+c_{i r} c_{j s}+c_{i s} c_{j r}\right) u_{i j} u_{r s} .
$$

This equation can be rearranged to give equation (7) in the main text of this paper. 